\section{Fatores associados ao uso de serviço de atenção pré-hospitalar por vítimas de acidentes de trânsito}

\author{
Factors associated with pre-hospital care in victims \\ of traffic accidents
}

\author{
${ }^{1}$ Faculdade de Medicina, \\ Universidade Federal de \\ Minas Gerais, Belo Horizonte, \\ Brasil. \\ 2 Gerência de Epidemiologia \\ e Informação, Secretaria \\ Municipal de Saúde de Belo \\ Horizonte, Belo Horizonte, \\ Brasil. \\ Correspondência \\ R. M. Ladeira \\ Departamento de Medicina \\ Preventiva e Social, \\ Faculdade de Medicina \\ Universidade Federal de \\ Minas Gerais. \\ Rua Itapema 305, apto. 701, \\ Belo Horizonte, $M G$ \\ 30310-490, Brasil. \\ roberto.marini@terra.com.br
}

\section{Abstract}

This was a cross-sectional study of all victims of traffic accidents in Belo Horizonte, Minas Gerais State, Brazil, admitted to the three largest public hospitals in the city from November 10 to December 14, 2003, to identify characteristics associated with the use of pre-hospital emergency treatment and investigate whether the time between the accident and hospital admission was shorter among these victims. The association between pre-hospital treatment and target variables was assessed by prevalence ratios obtained from Poisson regression. Among 1,564 victims, 778 (49.7\%) were transported in vehicles with pre-hospital treatment. Pre-hospital treatment was less common for bicyclists and pedestrians. The prevalence ratio was higher among victims with more severe injuries (AIS $=2$ and AIS $\geq 3$ ), older victims (30-39 years, 40-49 years, $\geq 50$ years), those who reported alcohol use, and when the time between accident and hospital admission was less than 60 minutes. According to the results, pre-hospital treatment is more frequent among severely injured victims and helps reduce the time between the accident and hospital admission.

Traffic Accidents; Emergency Medical Services; Prehospital Emergency Care
Roberto Marini Ladeira 1,2

Sandhi Maria Barreto 1

\section{Introdução}

$\mathrm{O}$ atendimento adequado e o tempo decorrido entre o acidente e a admissão hospitalar é um fator extremamente relevante para reduzir a mortalidade das vítimas de lesões produzidas por acidentes e violências. A primeira hora (golden hour) após a ocorrência de uma lesão traumática é considerada o tempo crítico para a instituição do tratamento que modificará o prognóstico, uma vez que até $40 \%$ dos óbitos ocorrem na fase pré-hospitalar do cuidado 1,2. Esse curto período de tempo é a margem de atuação do serviço de atenção pré-hospitalar, que visa a retirar a vítima de lesão traumática de forma rápida e segura do local do evento e levá-la ao local onde receberá o tratamento mais adequado ${ }^{3}$.

Prestar um cuidado adequado a essas vítimas pressupõe a existência de um sistema de atendimento de urgência que inclua um serviço de atenção pré-hospitalar articulado a hospitais com crescentes níveis de complexidade 4,5. A proposta e organização de serviços dessa natureza cresceu durante as guerras do século XX e fez cair substancialmente o tempo de remoção das vítimas até o atendimento definitivo, de 4 horas na Segunda Guerra Mundial, para 27 minutos na guerra do Vietnã 5. A partir daí, esta experiência é generalizada, especialmente para vítimas de lesões por causas externas em grandes centros urbanos. 
No Brasil, o serviço de atendimento pré-hospitalar começou a se desenvolver no início dos anos 90, voltado principalmente para o atendimento de vítimas de lesões traumáticas e foi implantado de forma heterogênea nas grandes cidades ${ }^{6}$. Em 2003, com o lançamento do Plano Nacional de Atendimento à Urgência e Emergência iniciou-se uma reformulação desse serviço, que passou a chamar-se Serviço de Atenção Móvel de Urgência (SAMU) abarcando também o atendimento às urgências clínicas 7 .

Na cidade de Belo Horizonte, com 2.300.000 habitantes, o serviço de atendimento pré-hospitalar foi implantado em dezembro de 1994 e, em pouco tempo já atendia a 500 chamados por mês, sendo 40,1\% dos atendimentos vítimas de acidentes de trânsito ${ }^{8}$. Apesar do crescente volume de atendimentos pelo serviço de atendimento pré-hospitalar, um percentual importante (52,9\%) de vítimas de acidentes de trânsito ainda é conduzido ao hospital por veículos particulares, táxis e, em casos de atropelamento, pelo motorista que atropelou 9 . Como se sabe, tal fato pode configurar risco de manuseio inadequado da vítima, com possibilidade de agravamento das condições clínicas e atraso no tratamento definitivo. O presente trabalho tem por objetivo conhecer as características dos acidentes e das vítimas de acidentes de trânsito que usaram o serviço de atendimento pré-hospitalar, e investigar se o uso deste serviço está associado a um menor tempo até o atendimento hospitalar.

\section{Métodos}

Estudo de corte transversal intitulado Pesquisa de Acompanhamento de Vítimas de Acidentes de Trânsito em Belo Horizonte e conduzido pela Secretaria Municipal de Saúde de Belo Horizonte e Empresa de Transporte e Trânsito de Belo Horizonte (BHTRANS) 9. O projeto foi desenvolvido em três hospitais públicos, principais instituições para atendimento às urgências no município em 2003 e que concentravam a grande maioria dos atendimentos hospitalares de vítimas de lesões por causas externas, funcionando como referência tanto para o serviço de atenção pré-hospitalar como para a população geral. No período de estudo, o serviço de atendimento pré-hospitalar de Belo Horizonte funcionava com uma central de regulação e 12 ambulâncias distribuídas pela cidade, sendo duas de suporte avançado de vida (com presença de um médico) e as demais com técnicos de enfermagem.

Foram elegíveis todos os indivíduos vítimas de acidentes de trânsito ocorridos no perímetro de Belo Horizonte e atendidos em um dos três hospitais de referência, no período de 10 de novembro a 14 de dezembro de 2003. A restrição a vítimas de acidentes ocorridos nos limites do Município de Belo Horizonte se justifica por ser esta a área de abrangência do serviço de atenção pré-hospitalar da cidade. O período foi escolhido por ser considerado típico, ou seja, sem longos feriados e antes do período de férias escolares.

Os dados foram coletados a partir de entrevista com a vítima ou seu acompanhante, mediante o preenchimento de questionário específico após assinatura do Termo de Consentimento Livre e Esclarecido. Os entrevistadores eram alunos do curso de Medicina previamente treinados para a aplicação do questionário e utilização das escalas de classificação de lesões. Foram obtidas informações sócio-demográficas das vítimas (sexo, idade, local de residência, uso de bebida alcoólica), características dos acidentes (tipo, local, veículo(s) envolvido(s), hora do dia, dia da semana) e características do transporte pré-hospitalar e transporte até o hospital (tipo, tempo entre o acidente e a chegada ao hospital e hospital de atendimento).

A descrição da localização e gravidade das lesões e os dados sobre a evolução foram retirados da ficha de atendimento hospitalar. A classificação da gravidade das lesões sofridas foi feita de acordo com NBR6061 - Gravidade das Lesões Sofridas por Vítimas de Acidentes de Trânsito, da ABNT (Associação Brasileira de Normas Técnicas), que incorpora a Escala Abreviada de Lesões (baseada na Abbreviated Injury Scale - AIS), variando de 0 a 6 conforme o aumento da gravidade das lesões 10 .

A variável dependente foi definida pelo uso ou não do serviço de atendimento pré-hospitalar. Os acidentados que usaram o serviço de atendimento pré-hospitalar foram comparados com aqueles conduzidos por outras formas de transporte com relação à distribuição das variáveis independentes: sexo, idade em cinco categorias (1-19 anos, 20-29, 30-39, 40-49 anos e $\geq 50$ anos), uso de bebida alcoólica (sim, não), hora do acidente (7:00 às 18:59 e 19:00 às 6:59), dia do acidente (dia útil, fim de semana), tipo de vítima (ocupante de automóvel, de motocicleta, de bicicleta e pedestre) e gravidade das lesões em três categorias (AIS 0/1, AIS 2 e AIS $\geq 3$ ). Embora não possa ser considerada como uma variável independente, foi também verificada a associação entre o tempo entre o acidente e a admissão hospitalar ( $<60$ minutos e $\geq 60$ minutos) e o uso do serviço de atendimento pré-hospitalar.

A associação entre uso do serviço de atendimento e as variáveis de interesse foi feita utilizando-se a razão de prevalências e o intervalo de confiança de $95 \%$, obtidos pela regressão de 
Poisson, adequada para estudos transversais. Todas as variáveis associadas ao transporte pelo serviço de atendimento pré-hospitalar com nível de significância $<0,20$ (exceto o sexo) foram consideradas na análise multivariada para verificar as variáveis associadas ao uso do serviço de atendimento pré-hospitalar. A modelagem final foi feita verificando-se em cada passo a significância estatística da inclusão da variável e a influência da mesma sobre a magnitude e direção das RP das demais variáveis no modelo. Foram mantidas no modelo final todas as variáveis que permaneceram associadas ao uso do serviço de atendimento pré-hospitalar ao nível de $\mathrm{p}<0,05$. Para a análise estatística utilizou-se o programa Stata 8.0 (Stata Corp., College Station, Estados Unidos)

O projeto de pesquisa foi aprovado pelos Comitês de Ética em Pesquisa (COEP) dos hospitais participantes.

\section{Resultados}

Foram admitidas 1.564 vítimas de acidentes de trânsito ocorridos no perímetro de Belo Horizonte entre 10 de novembro e 14 de dezembro de 2003. A maioria das vítimas foi atendida no hospital considerado a principal referência para atendimento ao trauma na cidade $(61,1 \%)$, era do sexo masculino $(73,3 \%)$, jovem $(65,9 \%$ com menos de 30 anos), residente em Belo Horizonte $(83,4 \%)$, não relatava uso de álcool $(82,3 \%)$, sofreu acidente durante o dia $(65,9 \%)$, em dia útil (60,8\%), chegou ao hospital em tempo inferior a 60 minutos após o acidente $(58,7 \%)$ e apresentou lesões leves $(75,9 \%)$. O maior percentual de vítimas era ocupante de motocicleta (36,3\%). Do total de vítimas, 778 (49,7\%) foram levadas aos hospitais pelo serviço de atendimento pré-hospitalar. Os demais foram transportados de outras formas, especialmente veículos particulares. Nas duas formas de transporte houve predomínio para o atendimento no hospital que é a principal referência para atendimento ao trauma em Belo Horizonte.

Todas as variáveis apresentaram baixo percentual de perdas, que variaram de $0 \%$ para o sexo até o máximo de 7,5\% para tempo decorrido entre o acidente e a chegada ao hospital. Não houve perda diferencial estatisticamente significativa entre os estratos das variáveis.

As vítimas transportadas pelo serviço de atendimento pré-hospitalar apresentaram média e mediana de idade mais elevadas (29,8 e 26,0 anos, respectivamente) do que as transportadas por outros meios (25,4 e 23,0 anos, respectivamente $-\mathrm{p}<0,001)$.
A Tabela 1 mostra a distribuição da população estudada, a prevalência de transporte pelo serviço de atendimento pré-hospitalar e as razões de prevalências brutas de acordo com as características demográficas e de atendimento das vítimas. Não houve diferença estatística entre os sexos com relação ao uso do serviço de atendimento pré-hospitalar. A prevalência de atendimento pelo serviço de atendimento pré-hospitalar aumentou diretamente com o aumento da idade, sendo a razão de prevalência para uso do serviço de atendimento pré-hospitalar 65\% maior para indivíduos com 50 ou mais anos quando comparados àqueles de $0 \mathrm{a}$ 19 anos. O socorro pelo serviço de atendimento pré-hospitalar também foi maior para os que relataram uso de álcool. O transporte pelo serviço de atendimento pré-hospitalar foi menor para todos os tipos de vítimas quando comparadas aos ocupantes de automóveis, mas esta diferença foi estatisticamente significativa apenas para ocupantes de bicicleta e pedestres. Verificou-se uma associação direta entre a prevalência de transporte pelo serviço de atendimento pré-hospitalar e a gravidade das lesões. Considerando-se como referência as vítimas com AIS $0-1$, a prevalência de transporte pelo serviço de atendimento pré-hospitalar foi $46 \%$ maior para vítimas com AIS = 2 e $85 \%$ maior para as com AIS $\geq 3$.

O transporte pelo serviço de atendimento pré-hospitalar também esteve associado estatisticamente a um menor tempo de chegada ao hospital. Mesmo sendo a variável com maior percentual de perdas de informação $(7,5 \%)$, a diferença nas perdas entre os que usaram o serviço de atendimento pré-hospitalar $(8,6 \%)$ e entre os que utilizaram outros meios de transporte $(6,4 \%)$ não foi estatisticamente significativa.

O transporte pelo serviço de atendimento pré-hospitalar não esteve associado à hora do acidente. Com relação ao período da semana, a maior prevalência de transporte pelo serviço de atendimento pré-hospitalar foi em acidentes no fim de semana.

As razões de prevalência ajustadas são apresentadas na Tabela 2. Permaneceram associados ao socorro pelo serviço de atendimento pré-hospitalar no modelo final a idade (faixas mais elevadas), o relato de uso de álcool, tipo de vítima (ocupantes de bicicleta e pedestres associados inversamente), a maior gravidade do acidente e a chegada ao hospital em tempo inferior a 60 minutos. 
Prevalência de transporte pelo serviço de atendimento pré-hospitalar e razões de prevalência (RP) brutas segundo características das vítimas de acidentes de trânsito. Belo Horizonte, Minas Gerais, Brasil, 2003.

\begin{tabular}{|c|c|c|c|}
\hline Variável & $n$ * & Prevalência (\%) & RP bruta (IC95\%) \\
\hline \multicolumn{4}{|l|}{ Sexo } \\
\hline Feminino & 418 & 48 & 1,00 \\
\hline Masculino & 1.146 & 50 & $1,05(0,94-1,18)$ \\
\hline \multicolumn{4}{|l|}{ Idade (anos) } \\
\hline 0-19 & 399 & 37,6 & 1,00 \\
\hline $20-29$ & 630 & 51,4 & $1,36(1,18-1,59)$ \\
\hline $30-39$ & 266 & 53,8 & $1,43(1,21-1,69)$ \\
\hline $40-49$ & 126 & 57,1 & $1,52(1,25-1,85)$ \\
\hline$\geq 50$ & 140 & 62,1 & $1,65(1,38-1,98)$ \\
\hline \multicolumn{4}{|l|}{ Uso de álcool } \\
\hline Não & 1.287 & 46,5 & 1,00 \\
\hline $\operatorname{Sim}$ & 238 & 65,1 & $1,40(1,26-1,56)$ \\
\hline \multicolumn{4}{|l|}{ Tipo de vítima } \\
\hline Ocupante automóvel & 303 & 60,7 & 1,00 \\
\hline Ocupante motocicleta & 568 & 53,2 & $0,87(0,78-0,99)$ \\
\hline Ocupante bicicleta & 256 & 34,4 & $0,56(0,47-0,69)$ \\
\hline Pedestre & 370 & 47,6 & $0,78(0,68-0,90)$ \\
\hline \multicolumn{4}{|l|}{ Gravidade da lesão (AIS) } \\
\hline $0-1$ & 1.221 & 44,1 & 1,00 \\
\hline 2 & 229 & 64,6 & $1,46(1,31-1,65)$ \\
\hline$\geq 3$ & 99 & 81,8 & $1,85(1,66-2,08)$ \\
\hline \multicolumn{4}{|l|}{ Intervalo de tempo (minutos) } \\
\hline$\geq 60$ & 598 & 43,0 & 1,00 \\
\hline$<60$ & 849 & 53,5 & $1,24(1,11-1,39)$ \\
\hline \multicolumn{4}{|l|}{ Hora do acidente } \\
\hline 19:00 às 6:59 & 505 & 52,3 & 1,00 \\
\hline $7: 00$ às $18: 59$ & 976 & 47,4 & $0,91(0,82-1,01)$ \\
\hline \multicolumn{4}{|l|}{ Período da semana } \\
\hline Dia de semana & 938 & 47,1 & 1,00 \\
\hline Fim de semana & 606 & 53,6 & $1,13(1,03-1,26)$ \\
\hline
\end{tabular}

AIS: Abbreviated Injury Scale

* As diferenças nos totais das categorias devem-se a perdas de informação.

\section{Discussão}

No presente estudo cerca de $50 \%$ das vítimas atendidas nos hospitais estudados foram transportadas pelo serviço de atendimento pré-hospitalar. Os resultados mostram que o transporte pelo serviço de atendimento pré-hospitalar foi maior em vítimas de acidentes de trânsito com lesões mais graves e que o mesmo está associado a um menor tempo entre a ocorrência do acidente e a chegada ao hospital. Essas são duas características essenciais de um serviço de atenção pré-hospitalar e figuram entre seus principais objetivos 11 .
As características das vítimas de acidentes de trânsito incluídas no presente estudo são similares às descritas em outros estudos de acidentes de trânsito no país, com preponderância de homens jovens 12,13 e de ocupantes de motocicletas 12,14 .

Estudos na área de acidentes de trânsito sugerem que os serviços de atenção pré-hospitalar apresentam resultados distintos com relação ao tempo de transporte. Entretanto, como a metodologia de aferição de tempo difere entre os diversos estudos, a comparabilidade dos resultados fica comprometida. Estudo realizado em Montreal, Canadá, mostrou que o tempo médio 
entre o acionamento do serviço e a chegada do paciente no hospital foi de 35,6 minutos 15 . Na Irlanda do Norte, o tempo médio de atendimento pré-hospitalar foi de 30,5 minutos 16. Em Monterey (México) e Seattle (Estados Unidos), os tempos médios de transporte pré-hospitalar foram de 73 e 31 minutos, respectivamente 1 .

No Brasil, Okumura \& Okumura 17 analisaram acidentes rodoviários no Estado de São Paulo e mostraram que o tempo médio entre o chamado do atendimento pré-hospitalar e a chegada ao hospital variou de 14 a 31 minutos. Whitaker et al. 18 encontraram que $81,3 \%$ das vítimas atendidas pelo SAMU no Município de São Paulo em 1991 tiveram um tempo de atendimento (entre o chamado e a chegada ao hospital) de até 40 minutos. Fernandes 19, analisando o serviço préhospitalar de Ribeirão Preto (São Paulo), mostrou uma média de 31 minutos entre o recebimento do chamado e a liberação da viatura. Em estudo realizado por Malvestio \& Souza 20 com acidentados nas vias expressas da cidade de São Paulo entre 1999-2000, o tempo total de atendimento variou de 37 a 46 minutos.

É importante ressaltar que, no presente estudo, o tempo contabilizado se refere ao período compreendido entre o momento do acidente e a chegada ao hospital, tanto para o serviço de atendimento pré-hospitalar quanto para os demais transportes. Ou seja, no caso do serviço de atendimento pré-hospitalar, ele representa a soma de vários tempos: entre a ocorrência do acidente e o chamado do pré-hospitalar, entre o recebimento do chamado e a saída da ambulância, entre a saída da ambulância de sua base até a chegada à cena, o tempo gasto na cena, e o tempo de deslocamento entre a cena e o hospital.

O uso do serviço de atendimento pré-hospitalar, entretanto, não garante que a vítima vai chegar ao hospital dentro da golden hour. Entre os participantes do presente estudo, por exemplo, $43 \%$ dos que levaram 60 minutos ou mais para chegar ao hospital foram transportados pelo serviço de atendimento pré-hospitalar. O tempo decorrido entre o evento e a entrada no hospital depende de vários fatores. A chegada do serviço de atendimento pré-hospitalar à cena do acidente, por exemplo, é fortemente influenciada pela distância do local do acidente, as condições de tráfego e a disponibilidade de ambulâncias. No caso deste trabalho, a informação sobre a hora do acidente foi obtida diretamente com a vítima ou com seus acompanhantes/transportadores. Ou seja, não dispomos da informação sobre a hora em que o serviço de atendimento pré-hospitalar foi acionado, o que permitiria estimar o tempo efetivamente levado desde a chamada do mesmo até a chegada ao hospital. Além disso, o
Tabela 2

Razão de prevalência (RP) ajustada de transporte pelo serviço de atenção pré-hospitalar conforme modelo logístico. Belo Horizonte, Minas Gerais, Brasil, 2003. ( $N=1.343)$.

\begin{tabular}{|c|c|c|}
\hline Variáveis & RP ajustada (IC95\%) & $\mathrm{p}$ \\
\hline \multicolumn{3}{|l|}{ Idade (anos) } \\
\hline $0-19$ & 1,00 & \\
\hline $20-29$ & $1,16(0,98-1,38)$ & 0,08 \\
\hline $30-39$ & $1,26(1,05-1,52)$ & 0,01 \\
\hline $40-49$ & $1,32(1,06-1,64)$ & 0,01 \\
\hline$\geq 50$ & $1,51(1,23-1,86)$ & 0,00 \\
\hline \multicolumn{3}{|l|}{ Uso de álcool } \\
\hline Não & 1,00 & \\
\hline Sim & $1,26(1,11-1,43)$ & 0,00 \\
\hline \multicolumn{3}{|l|}{ Tipo de vítima } \\
\hline Ocupante automóvel & 1,00 & \\
\hline Ocupante motocicleta & $0,89(0,78-1,03)$ & 0,13 \\
\hline Ocupante bicicleta & $0,64(0,52-0,79)$ & 0,00 \\
\hline Pedestre & $0,73(0,62-0,87)$ & 0,00 \\
\hline \multicolumn{3}{|l|}{ Gravidade da lesão (AIS) } \\
\hline $0-1$ & 1,00 & \\
\hline 2 & $1,47(1,30-1,65)$ & 0,00 \\
\hline$\geq 3$ & $1,76(1,53-2,03)$ & 0,00 \\
\hline \multicolumn{3}{|c|}{ Intervalo de tempo (minutos) } \\
\hline$\geq 60$ & 1,00 & \\
\hline$<60$ & $1,22(1,09-1,36)$ & 0,00 \\
\hline
\end{tabular}

AIS: Abbreviated Injury Scale.

cálculo do tempo decorrido entre a ocorrência do acidente e a chegada ao hospital, feito a partir do relato da vítima ou seu acompanhante, pode ocasionar importantes erros na precisão da informação.

Um achado relevante é o fato de haver uma associação negativa entre o transporte pré-hospitalar de pedestres ou de ocupantes de bicicletas (vítimas que em geral apresentam lesões mais graves) em relação aos ocupantes de automóveis, considerados menos vulneráveis 13 . Possíveis explicações para tal fato podem ser a maior probabilidade de socorro pelo atropelador, no caso do pedestre, e o maior acesso a telefone móvel pelos ocupantes de veículos automotores. Como o modelo final considerou a gravidade da lesão, é provável que a maior letalidade dos acidentes envolvendo pedestres no local e sua remoção diretamente para o Instituto Médico Legal, seja uma explicação para o resultado encontrado.

$\mathrm{O}$ achado de um gradiente de prevalência de transporte pelo serviço de atendimento préhospitalar de acordo com o aumento da faixa etária pode indicar um critério de decisão de 
atendimento, que considera a idade mais elevada como um fator independentemente associado à maior ocorrência de complicações, permanência hospitalar e mortalidade em vítimas de lesões traumáticas conforme já relatado na literatura 21 .

A associação entre uso de bebida alcoólica e transporte pelo serviço de atendimento pré-hospitalar merece cautela uma vez que não houve comprovação laboratorial dos relatos obtidos. A análise estratificada dos acidentes por gravidade (AIS $\geq 3$ e AIS < 3) confirma a associação entre relato de uso de álcool e maior uso do serviço de atendimento pré-hospitalar, sugerindo que a mesma independe da gravidade. Uma hipótese seria a maior tendência a chamar o serviço de atendimento pré-hospitalar para vitimas alcoolizadas, devido, por exemplo, à maior dificuldade em discernir as manifestações decorrentes do uso do álcool de sintomas neurológicos mais graves 22 .

O maior volume de atendimentos no hospital especializado no atendimento ao trauma, tanto para o serviço de atendimento pré-hospitalar como para a população em geral, é condizente com sua condição de principal referência para o atendimento de vítimas de traumatismos por acidentes de trânsito em Belo Horizonte.

O percentual de vítimas transportadas pelo serviço de atendimento pré-hospitalar ainda é pequeno se compararmos aos $96 \%$ observados em Monterey e Seattle 1 ou com os $65 \%$ relatados em Cuernavaca (México) 23. Não foram localizados dados nacionais publicados sobre o percentual de vítimas transportadas pelo serviço de atendimento pré-hospitalar, estudadas a partir do atendimento hospitalar. Os estudos identificados sobre o assunto analisaram o serviço de atendimento pré-hospitalar na perspectiva exclusiva das características das vítimas atendidas por este serviço 12,17,18,19,20.

Apesar da importância da questão de acesso ao serviço de atendimento pré-hospitalar, o mesmo não foi analisado no presente estudo por ausência de informações. Ou seja, não sabemos se as vítimas que chegaram aos hospitais por outros meios solicitaram o serviço de atendimento préhospitalar. Também não foi investigado, entre as vítimas atendidas pelo serviço de atendimento pré-hospitalar, se o atendimento foi realizado por uma unidade básica ou de suporte avançado e se foram realizados procedimentos terapêuticos na cena do acidente ou durante o transporte, o que poderia, eventualmente, aumentar o tempo de chegada ao hospital.

Novos estudos sobre o assunto são necessários para avaliar a cobertura, acesso e qualidade dos serviços de atenção pré-hospitalar no Brasil, levando-se também em consideração a grande expansão destes serviços em nosso país, assim como a mudança do perfil de atendimento ocorrida a partir da implantação do SAMU. 


\section{Resumo}

Em um estudo de corte transversal foram avaliadas as características das vítimas de acidentes de trânsito ocorridos em Belo Horizonte, Minas Gerais, Brasil, com o objetivo de conhecer as características dos acidentes e das vítimas que usaram o serviço de atendimento pré-hospitalar, e investigar se o uso deste serviço está associado a um menor tempo até o atendimento hospitalar. Participaram do estudo todas as 1.564 vítimas de acidente de trânsito atendidas nos três maiores hospitais públicos de referência para emergência na cidade entre 10 de novembro e 14 de dezembro de 2003. As associações foram investigadas utilizando-se razões de prevalência obtidas por regressão de Poisson. Os resultados mostraram que 49,7\% das vítimas usaram o serviço de atendimento pré-hospitalar, sendo a utilização menor entre ocupantes de bicicleta e pedestres. A gravidade (AIS = 2 e AIS $\geq 3$ ), idade (30-39 anos, 40$49, \geq 50$ anos), relato de uso de álcool e tempo de admissão hospitalar $<60$ minutos estiveram associados ao transporte pelo serviço de atendimento pré-hospitalar. O uso do serviço de atendimento pré-hospitalar foi maior entre vítimas mais graves e esteve associado a um tempo menor de chegada até o hospital, características consideradas essenciais para um serviço de atenção pré-hospitalar.

Acidentes de Trânsito; Serviços Médicos de Emergência; Atendimento de Emergência Pré-Hospitalar

\section{Referências}

1. Mock CN, Jurkovich GJ, nii-Amon-Kotei D Arreola-Risa C, Maier RV. Trauma mortality patterns in three nations at different economic levels: implications for global trauma system development. J Trauma 1998; 44:804-14.

2. Trunkey DD. Trauma. Sci Am 1983; 249:28-35.

3. Harrington DT, Connoly M, Biffl WL, Majercik SD, Cioffi WG. Transfer times to definitive care facilities are too long - a consequence of an immature trauma system. Ann Surg 2005; 241:961-8.

4. Arreola-Risa C, Mock CN, Padilla D, Cavazos L, Maier RV, Jurkovich GJ. Trauma care systems in urban Latin America: The priorities should be prehospital and emergency room management. J Trauma 1995; 39:457-62.

5. Nathens AB, Brunet FP, Maier RV. Development of trauma systems and effect on outcome after injury. Lancet 2004; 363:1794-801.

6. Brasil. Portaria $n^{\circ}$. 814 de 1 de junho de 2001. Institui as diretrizes de regulação das urgências e normatiza os serviços de atendimento pré-hospitalar móvel. Diário Oficial da União 2001; 4 jun.

\section{Colaboradores}

R. M. Ladeira participou do planejamento do estudo, supervisão do trabalho de campo, análise dos dados e redação do manuscrito. S. M. Barreto participou da análise dos dados e redação do manuscrito.

\section{Agradecimentos}

Celeste de Souza Rodrigues - Secretaria Municipal de Saúde de Belo Horizonte, João Pereira Carvalho e Ilma Maria de Paula - Empresa de Transporte e Trânsito de Belo Horizonte, que também coordenaram a realização do projeto Pesquisa de Acompanhamento de Vítimas de Acidentes de Trânsito em Belo Horizonte em 2003. Este projeto foi financiado pela Secretaria Municipal de Saúde de Belo Horizonte e Empresa de Transporte e Trânsito de Belo Horizonte.
7. Portaria $n^{\circ} .1864$ de 29 de setembro de 2003. Institui o componente pré-hospitalar móvel da Política Nacional de Atenção às Urgências. Diário Oficial da União 2003; 5 out.

8. Magalhães Jr. HM. Urgência e emergência - a participação do município. In: Campos CR, Malta DC, Reis AT, Santos AF, Merhy EE, organizadores. Sistema Único de Saúde em Belo Horizonte - reescrevendo o público. São Paulo: Xamã Editora; 1998. p. 265-86.

9. Secretaria Municipal de Saúde de Belo Horizonte. Saúde em trânsito: pesquisa de acompanhamento de vítimas de acidentes de trânsito em Belo Horizonte, 2005. http://www.opas.org.br/ informacao/UploadArq/transito.pdf (acessado em 28/Mar/2007)

10. Associação Brasileira de Normas Técnicas. Gravidade das lesões sofridas por vítimas de acidentes de trânsito - NBR 6061. São Paulo: Associação Brasileira de Normas Técnicas; 1980. 
11. Sikka N, Margolis G. Understanding diversity among prehospital care delivery systems around the world. Emerg Med Clin North Am 2005; 23:99114

12. Bastos YGL, Andrade SM, Soares DA. Características dos acidentes de trânsito e das vítimas atendidas em serviço pré-hospitalar em cidade do Sul do Brasil, 1997/2000. Cad Saúde Pública 2005; 21:81522.

13. Marson AC, Thomson JC. The influence of prehospital care on motor vehicle crash mortality. J Trauma 2001; 50:917-21

14. Andrade SM, Mello-Jorge MHP. Características das vítimas por acidentes de transporte terrestre em município da região Sul do Brasil. Rev Saúde Pública 2000; 34:149-56.

15. Sampalis JS, Lavoie A, Willians JI, Mulder DS, Kalina M. Impact of on-site care, prehospital time, and level of in-hospital care on survival in severely injured patients. J Trauma 1993; 34:252-61.

16. McNicholl BP. The golden hour and prehospital trauma care. Injury 1994; 25:251-4.

17. Okumura M, Okumura CH. Atendimento pré-hospitalar de acidentados de tráfego rodoviário: experiência brasileira. Rev Hosp Clin Fac Méd São Paulo 1994; 49:45-8.
18. Whitaker IY, Gutiérrez, MGR, Koizumi, MS. Gravidade do trauma avaliada na fase pré-hospital. Rev Assoc Med Bras 1998; 44:111-9.

19. Fernandes RJ. Caracterização da atenção pré-hospitalar móvel da Secretaria de Saúde do Município de Ribeirão Preto-SP [Dissertação de Mestrado]. Ribeirão Preto: Escola de Enfermagem de Ribeirão Preto, Universidade de São Paulo; 2004.

20. Malvestio MAA, Souza RMC. Suporte avançado à vida: atendimento a vítimas de acidentes de trânsito. Rev Saúde Pública 2002; 35:584-9.

21. Morris JA, MacKenzie EJ, Damiano AM, Bass SM. Mortality in trauma patients: the interaction between host factors and severity. J Trauma 1990; 30:1476-82.

22. Gurney JG, Rivara FP, Mueller BA, Newell DW, Copass, MK, Jurkovich GJ. The effects of alcohol intoxication on the initial treatment and hospital course of patients with acute brain injury. J Trauma 1992; 33:709-13.

23. Hidalgo-Solórzano EC, Híjar M, Blanco-Muñoz J, Kageyama-Escobar ML. Factores associados com la gravedad de lesiones ocorridas en la vía pública en Cuernavaca, Morelos, México. Salud Pública Méx 2005; 47:30-8.

Recebido em 26/Abr/2007

Versão final reapresentada em 29/Jun/2007 Aprovado em 07/Ago/2007 